\title{
The Dimensioning of Non-Token-Bucket Parameters for Efficient and Reliable QoS Routing Decisions in Bluetooth Ad Hoc Network
}

\author{
Halabi Hasbullah and Mahamod Ismail \\ Universiti Teknologi PETRONAS, \\ Universiti Kebangsaan Malaysia, \\ Malaysia
}

\section{Introduction}

\subsection{Bluetooth applications and its technical issues}

For specific applications, such as in Wireless Personal Area Network (WPAN), Bluetooth ad hoc network can be suitably deployed as a substitution for other technologies to provide the last meter connectivity solutions. In a WPAN, as specified in IEEE 802.15 Specification, nearby devices can be connected together for short-range communications to form a personal networking setup, for instance between a hand phone and an ear phone, an access point and a PDA, a GPS receiver and a navigator, etc. The other short-range technologies, such as Wireless Sensor Network (WSN), ZigBee, and Radio Frequency IDentification (RFID) may not be able to create such personal networking capability. Bluetooth communications technology was originally designed and intended to replace the cable connectivity between these nearby devices. Now, all the devices within the WPAN are neatly and seamlessly connected together without cable cumbersome, as well as providing mobility support to end users. To some extent, roaming facility is provided to allow a user to move around from one coverage area to another. This roaming service may be applicable in a museum or shopping mall where information about items or products is transmitted transparently to users' or clients' mobile devices, while they are on the move. In this way, the users would be more informative about the services and products offering around them.

However, Bluetooth ad hoc network is constrained by limited resources due to its lowpower and short-range communication capability, as described by (Haartsen, 1998). The smallest networking unit of Bluetooth, called piconet, can support up to 8 active mobile devices at a time. A greater number of mobile devices in vicinity can only be supported by creating multiple piconets, which now a scatternet that interconnects multiple piconets is developed. Additionally, its communications range can only cover to a maximum of 100 meters and beyond this range multi-hop communication using multiple relay devices is required. This is in contradiction to WLAN, WiFi and WiMAX setups, where hundreds of mobile devices are handled simultaneously and wider coverage area is obtained by using only a single cell. However, with no exception, Bluetooth ad hoc network is also carrying multimedia, interactive, and real-time data, and the demands for transporting these data 
types over the network is keep increasing every day. Simply, Bluetooth is a limitedresources network type but to accommodate growing and demanding applications.

Figure 1 depicted a typical Bluetooth scatternet topology, created by a number of interconnected piconets. A piconet is made up of one master node and a maximum of 7 active slave nodes, where the master node is in control of all the slave nodes. Two slave nodes in a piconet cannot directly communicate with each other, but communication is allowed after passing through the master node that controls them. A node that resides in the overlapping coverage area between two or more scatternets is called a bride or relay node. A node may become a slave node in a piconet and at the same time acts as a master node in another piconet. In this case, role switching is required at different time instances to change the role from a master node to a slave node, and vice versa. As a result, switching time is required, thus delay transmission time is introduced.

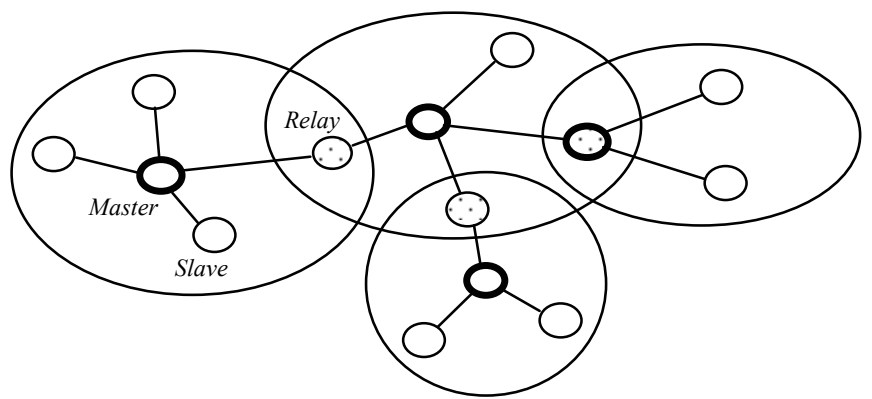

Fig. 1. A typical Bluetooth ad hoc network with a topology of scatternet

A relay node that connects two piconets is likely to behave as a router node in the scatternet, forwarding packets from one piconet to another piconet in the topology. Hence, a router node is in the position of making decision to select one forwarding link from the available outgoing links of that node, based on certain decision criteria. For reliable packets transmission, redundant links may be selected with additional costs. The selected link not only has to satisfy the demand for the required resources, but also to provide certain degree of service guarantee to the requested application, and ultimately to the end users.

Hence, one could guess that the basic technical challenge that appeared in the Bluetooth ad hoc network is its limited communication capabilities, derived from its limited transmission power that it can emit, limited distance that it can cover, and limited device number that it can handle simultaneously. These limitations however, should not hinder the Bluetooth network from providing similar services as that of the other wireless networks have offered. Considering the limitations, therefore providing Quality of Service (QoS) in this network has been a major issue. One of such QoS issues is efficient and reliable routing in the network, by which a level of service guarantee must be provided for every single packet sent. The efficiency can be interpreted as consuming as much low power energy as possible to prolong the network lifetime, i.e. the lesser energy consumed the longer lifetime of the overall network. Reliability can be defined as having low or no packet lost probability in each transmission in order to uphold the data integrity. High packet lost probability is a reflection of unreliable transmissions. To achieve these two QoS goals, every single device in the Bluetooth network, regardless of their role of whether master or slave node, is expected to use the available but 
limited resources wisely during the course of routing and transmitting. However, their contributions for the transmission efficiency and reliability are determined by several internal and external factors, such as bursty traffic pattern, channel condition, etc.

\subsection{Bluetooth and traffic characterization}

The characteristic of today's traffic is naturally bursty (Leland et al., 1994). Real-time and interactive applications from web browsing, audio/video conferencing, video-on-demand, forecasting, sensoring, on-line transactions, and multiparty games are generating bursty traffics. Traffic is considered as bursty when there is an unpredictable fluctuations of similar data blocks patterns that appeared at any point of times during its transmission. In its simplest form, burst is defined by (Handle et al., 1996) as a ratio between peak and mean bit rate. In many cases, burst can be triggered from rare events. For example, when several large size file transfers is detected as compared to average size file transfers, several intervals of too long packets arrival times as compared to average packets arrival times, etc. In another form, burst in a traffic stream is determined by its high variability over a time series.

Bursty traffic, as described by (Park et al., 1996), is known to associate with self-similar property. Also, as had been determined by (Leland et al., 1994), bursty traffic induced a direct impact on network performance, which may be in the form of unreliable routing decision at router node. The variability in the input traffic does not allow network performance to be accurately measured, i.e. performance consistency does not appear at different time series. Additionally, the parameters that contribute to the inconsistency may not be identifiable. As a result, network control software could not be designed to effectively and seamlessly manage the router nodes, and ultimately the network system may fail to perform as expected. If this happened, QoS is hard to achieve because the traffic's characteristic cannot be accurately described to Resource Manager for reservation and allocation of resources when routing decision is about to be made at a router node. Obviously, efficiency, reliability, and service guarantee in the routing function will not be achieved in the network. Simply, burst of traffic has an impact on how a mobile device in the scatternet makes its routing decisions, as well as on the overall performance of the Bluetooth network. On other factor, channel quality of a link that connects between two adjacent mobile nodes may also affect the network performance, particularly when QoS routing decision is to be made by one of these nodes. High bit error rate on the selected link may lead to low transmission quality, and high packet lost probability may result in unreliable connectivity.

Hence, if some parameters of the source bursty traffic and the error-prone wireless channel can be accurately dimensioned for resource reservation and allocation to Resource Manager, a better level of QoS can then be granted to user applications. For this purpose, a trafficdescriptor is required to describe the characteristics of the input traffic and the current wireless channel condition to the Resource Manager for appropriate QoS requirements. In other words, by having a traffic-descriptor, the QoS demands for a specific routing task can now be more accurately stated. In this way, the achievement toward QoS provisioning in Bluetooth ad hoc network is more promising than before and thus, promoting the Bluetooth technology and its applications to a greater height. Token-Bucket scheme can be used to produce such traffic-descriptor.

The development of a traffic-descriptor so far however, had only considered the Token-Bucket parameters, while the uses of non-Token-Bucket parameters, which they may contribute 
significantly to QoS provisioning, have been omitted. The prime reason for inclusion of the non-Token-Bucket parameters is to meet the various and flexible QoS demands, while TokenBucket can only provide standard and fixed parameters with limited usage. Importantly, a parsimonious traffic-descriptor is required, where only least parameters are used in the trafficdescriptor, but it has the ability to efficiently and reliably providing QoS routing decisions at a router node. Hence, if the resource-limited Bluetooth ad hoc network is to be used effectively in supporting the WPAN implementation, a parsimonious traffic-descriptor that also contained the non-Token-Bucket parameters must be developed.

Figure 2 illustrates router nodes of $i$ and $k$, at which decisions are to be made to select one forwarding link from the available outgoing links to forward their received packets to the next node, based on certain decision criteria (e.g. the bit rate of the link and length of the link). To execute this routing task, a mapping between the available bit rate of the link and the requested bit rate from the application is performed, and see whether or not this request can be fulfilled. The selected forwarding link not only has to satisfy the demand of the bit rate, but also to provide certain degree of efficiency and reliability for the forwarding task. However, in many nowadays complex and sophisticated application scenarios, there are multiple QoS demands that need to be satisfied. For this reason, the selected forwarding link may only be able to provide an optimal solution to the QoS routing function. Note that a complete route is made up of a set of links. Therefore, a set of routing decisions are to be made along a complete route that connects a pair of source node and sink node in the scatternet topology. Hence, the final result shall be exhibited through an optimal use of the limited resources over the complete route.

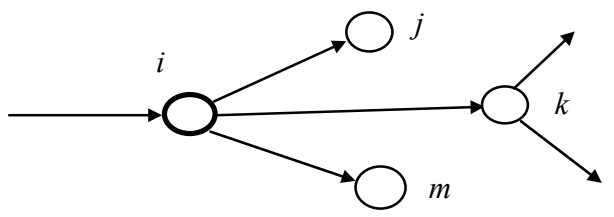

Fig. 2. Routing decision at router nodes $i$ and $k$

Therefore, to describe the characteristics of the source traffic and forwarding link for resource reservation and allocation during routing decision, a traffic characterization process is required, such that resources are appropriately reserved and allocated. To support the characterization process, a mathematical model must be developed, which will include the parameters of input traffic, channel condition, and others that are expected to have direct impact on the routing performance, as well as on the network QoS provisioning.

\subsection{The issues in traffic characterization}

However, in developing an effective traffic-descriptor, a problem has appeared during the formulation of a mathematical model that could parsimoniously incorporate only least possible parameters, but well describing the application's QoS requirements. Specifically, how the non-Token-Bucket parameters, which may contribute significantly to the efficiency and reliability of a routing function can be embedded into the traffic-descriptor. This is critical for Bluetooth ad hoc network for two very basic reasons. First, most of the time, only a single link is provided between any two communicating devices, thus no option available to select any other efficient and reliable links. This situation of only-one-link availability is 
created in Bluetooth network because of the master-driven communication approach, where nearby slave nodes in a piconet cannot directly communicate, except through a master node. Second, Bluetooth is a resource-limited network provided with low-power and short-range communication capabilities, and hence the available resources must be used efficiently. Hence, with parsimonious traffic-descriptor, the processing time would be faster and the energy usage would be lower. In short, Bluetooth ad hoc network has many constraints with respect to its ability to fulfill the ever-increasing demands for the QoS guarantees.

Therefore, the objective of this work is to develop a parsimonious traffic-descriptor that could incorporate the non-Token-Bucket parameters, together with the standard TokenBucket parameters so that an efficient and reliable QoS routing decisions could be made at a router node. This requires the task of dimensioning the traffic-desriptor's parameters, i.e. the determination of the parameters and analyze their impacts in the routing decision-making processs at a router node. In order to dimension the parameters, characterization process on the environment of the routing function is required.

For the purpose of producing the such traffic-descriptor, a mathematical model that measures the burstiness level $\alpha$ and the degree of self-similarity $H$ in a traffic stream, as well as the channel quality (via bit error rate) must be developed. Using this deterministic information, the QoS provisioning shall be more guaranteed. However, the exact procedures for resource reservation and allocation are not within the scope of this research work; it shall be given to Resource Manager to manage the complete procedures. Thus, this work is limited to only producing traffic-descriptor for Resource Manager to use.

To verify the usefullness of the developed parsimonious traffic-descriptor in providing supports for QoS routing in Bluetooth ad hoc network, a Matlab simulated router node was developed to measure its performance. It was found that a parsimonious traffic-descriptor could be generated with a promising performance. That is to say, with only few parameters, the traffic-descriptor has been able to meet the required QoS routing demands. With the result, it has promoted Bluetooth ad hoc network to a higher level of usefulness and practical applications, particularly in solving the last-meter connectivity issues.

The rest of this chapter is organized as follows. Section 2 discusses self-similar property that appeared in a traffic burst and the use of Token-Bucket scheme in characterizing the burst. Section 3 explains the methodology to derive a traffic-descriptor. Results and analysis on the performance of the developed traffic-descriptor in supporting QoS routing decision at a router node are presented in Section 4. Finally, conclusion and future work are made in Section 5.

\section{Related works}

\subsection{Self-similar property in the burst}

It has been a long belief that network traffic pattern is following Poisson distribution. However, in actual fact it has been proved that it was only applicable to speech data of the telephony system. Discovery by (Leland et al., 1994) has provided evidence that the interarrival times for bursty traffic in local area network (LAN) is actually following heavy-tailed distribution of power law. Their study on Ethernet LAN traffics from 1989 to 1992 has established that fractal (or self-similar) property in a traffic stream could not any longer be captured by conventional traffic model. Supported by (Paxson, 1995), it was confirmed that the packets' inter-arrival times have deviated away from exponential distribution, but following a heavy-tailed distribution. Heavy-tailed distribution is characterized by a slowly decaying tail of the hyperbolic graph to infinity. Additionally, a work by (Beran, 1994) has 
determined that variable bit rate of MPEG video traffic is associated to self-similarity, which is common property for the bursty traffics.

As determined by the Bluetooth Specifications v1.0B, (1999), Bluetooth network implements Segmentation and Reassembly (SAR) protocol at L2CAP layer, by which long message blocks received from upper layers are segmented into smaller packets types of DMx or DHx (M - Medium, H - High, $x=1,3,5$ slots). Since the network is also handling bursty input traffic, it is anticipated that the SAR protocol execution on MPEG video data might produce the same heavy-tailed distribution with respect to some of its features.

To describe heavy-tailed distribution and self-similar property, second order statistics is required. Heavy-tailed distribution is defined by (Crovella \& Lipsky, 1997) as follows. Let $X$ be a random variable with cumulative distribution function (cdf) of $F(x)=P(X \leq x)$ and complementary cumulative distribution function (ccdf) of $\bar{F}(x)=1-F(x)=P(X>x)$. A distribution $F(x)$ is said to be heavy-tailed if

$$
\bar{F}(x)=P(X>x) \sim c x^{-\alpha}
$$

when $x \rightarrow \infty$ for positive $c$ value and $0<\alpha<2$. In other words, a distribution is heavy-tailed if the ratio of $P(X>x) / x^{-\alpha}$ is approaching 1 when $x \rightarrow \infty$ for $\alpha>0$. The asymptotic form of the distribution is following a power law distribution. One of the simplest heavy-tailed distribution is Pareto distribution with probability distribution function (pdf) of the form $f(x)=\alpha k^{\alpha} x^{-\alpha-1}$, where $\alpha>0,0<k \leq \mathrm{x}$. Accordingly, the distribution is respectively having cdf of

and ccdf of

$$
F(x)=P(X \leq x)=1-(k / x)^{\alpha}
$$

$$
\bar{F}(x)=P(X>x)=(k / x)^{\alpha}
$$

where $\alpha$ is a shape parameter and $k$ is a scale parameter.

The mean for Pareto distribution is $\mu=\alpha k /(\alpha-1)$ and the variance is $\sigma^{2}=\alpha k^{2} /(\alpha-1)^{2}(\alpha-2)$. If $\alpha<1$, the distribution would have infinite mean; if $\alpha<2$, the distribution would have infinite variance; if $1<\alpha<2$, it would has finite mean and infinite variance; and if $\alpha \geq 2$, both mean and variance are finite. In general, if its variance is infinite, then $X$ would associate to high variability in its distribution.

One important property of the heavy-tailed distribution is that it is self-similar, as have been proved by (Leland et al., 1994) and supported by (Crovella \& Bestavros, 1999). Additionally, as claimed by (Taqqu et al., 1997), superimposition of several independent of ON/OFF heavy-tailed traffic sources is just enough to produce a self-similar traffic stream.

Self-similarity is defined by (Fernandes et al., 2003) as follows. Let $X(t)$ be a wide-sense stationary time series with mean $\mu$, variance $\sigma^{2}$, and autocorrelation function $\rho(\tau)$. Let $X^{m}(t)$ be a newly derived time series from $X(t)$ by averaging a number of $m$ non-overlapping block sizes. Its aggregated series is

$$
X^{\mathrm{m}}(t)=\left(\mathrm{m}^{-1}\right)\left(X_{\mathrm{tm}-\mathrm{m}+1}+X_{\mathrm{tm}-\mathrm{m}+2}+\ldots+X_{\mathrm{tm}}\right)
$$

and $\rho^{\mathrm{m}}(\tau)$ is its autocorrelation function. Process $X(t)$ is said to be self-similar if

$$
\rho^{n}(\tau)=\rho(\tau) \text { for } m=1,2,3, \ldots
$$


A work by (Park et al., 1996) has proved that self-similar property has a direct impact on network performance. Also, as identified by (Leland et al., 1994), if it is known that the source traffic is bursty, two definite consequences will occur: the increase in buffer requirement and the longer delay experienced.

To characterize the burst property, it is important to identify the level of burstiness $\alpha$ and the degree of self-similarity $H$ in the source traffic stream. The relationship between $\alpha$ and $H$ for Pareto distribution has been derived by (Leland et al., 1994), which is expressed as $H=(3-\alpha) / 2$. As stated by (Hadzi-Velkov \& Garrilovska, 1999), $\alpha$ is an indicator of the burstiness level in a traffic stream. For the source traffic to have a heavy-tailed distribution, an interval of $1<\alpha<2$ must be obtained, where $\alpha \rightarrow 1$ indicates too bursty traffic. On the other hand, to measure the degree of self-similarity, an interval of $0.5 \leq H<1$ is to be obtained. $H \rightarrow 1$ indicates a high degree of self-similarity.

Combined with some other characteristics of the traffic stream and/or system, such as packets' efficiency and channel quality, the QoS requirements of an application can be described to Resource Manager in a much more accurate manner. As have been discussed, it is stated in a traffic-descriptor. In this way, much better resource reservation and allocation could be made, and deterministic network performance could be measured and obtained. Then, QoS can be granted to user applications with higher degree of confidence.

\subsection{Token-Bucket scheme}

There have been many works in the literature describing on the use of Token-Bucket (TB) scheme for traffic regulation, which the usage was based only on its own basic parameters. Work by (Norashidah \& Norsheila, 2007) has improvised the TB by including a fuzzy logic component, resulting in a fuzzy logic TB predictor that has the ability to adapt its token rate based on actual traffic requirements. In this way, the actual bandwidth requirement can be predicted and feedback is relayed to Admission Control mechanism. In order to characterize the bursty input traffic, TB scheme may be used in a 2-in-1 combined function as proposed by (Procissi et al., 2001). The first function is to regulate the arriving burst to a more controllable and deterministic form of traffic flow. The second function is to characterize the incoming self-similar traffic so that the conbtributing parameters could be identified and measured. Subsequently, a traffic-descriptor is produced.

Typically, a traffic-descriptor is expressed as $(\rho, b)$, where $\rho$ is the token rate and $b$ is the bucket size, and both are the basic TB parameters. Papers by (Li, 2002) and (Glasmann et al., 2000) have elaborated the production of a traffic-descriptor using TB scheme. However, work by (Yang, 2000) has suggested a traffic-descriptor of the form ( $\rho$, unlimited), by which the bucket size $b$ can be as large as possible. However, as confirmed by queuing theory, the larger bucket size the longer processing delay experienced by the packets in the queue and in other subsequent processing tasks. This claim is supported by a scheduling work of (Garroppo et al., 2001). In summary, study by (Procissi et al., 2001) could be the best piece of work that has taken into account the self-similar property of the source traffic for QoS routing decisions at a router node, by which the other previous works did not.

However, the critical issue in QoS provisioning in Bluetooth ad hoc network is to find an appropriate probabilistic model for the source traffic, in particular if stochastic approach is used as described by (Valaee \& Gregoire, 2005). As determined by (Li, 2002), the production of a traffic-descriptor is application-dependent and case-sensitive. Furthermore, as stated by (Glasmann et al., 2000) that until today, a standard procedure to produce a traffic-descriptor 
is still none existence. Therefore, the development of a parsimonious traffic-descriptor for Bluetooth ad hoc network is still an open issue and research opportunities on it are wide open. Parsimonious is referred to as having only least possible parameters but well describing the system in question, whilst achieving a level of efficiency and reliability in routing. This is because the more parameters to handle, the more resources are required, which may not lead to QoS provisioning. Therefore, parsimonious is very much needed in Bluetooth ad hoc network since it has only limited resources to offer.

However, the TB scheme alone may not be able to completely describe the source traffic and the network characteristics. Therefore, some other components are required to work with the $\mathrm{TB}$, by which these components will provide the necessary additional non-TB parameters. The expected result shall be of a more accurate reservation and allocation of network resources from users applications for each routing decision made at a router node.

\section{Methodology}

\subsection{The system model}

In a Bluetooth scatternet topology, the master node is usually the one that makes most routing decisions. However, depending on the established topology, a slave node may also be in the position of making the same routing decisions, especially when the node is a relay/bridge node connecting two or more piconets. The decision must not only accomodating the constrainted resources but also meeting the QoS demands of the applications in terms of its efficiency and reliability. For this reason, only optimal routing solution may be achieved at any point of routing instances. To achieve this QoS routing requirement, Token Bucket (TB) approach is suggested to be used to handle the burst issue. In this work, TB smoothing scheme is initially used to smooth out the bursty traffic input, as described by (Yang, 2000). This is done by applying buffer queue for the received burst, while the non-burst can easily be processed at normal rate. With the smoothed, shaped, and controlled traffic, the network performance could be measured and its performance parameters could be identified. In this way, controll software could then be developed to handle the burtsy traffic in an automated manner. However, the function provided by the standard TB with its basic parameters, will not be able to handle the issue of self-similar property that come together with the burst. The basic function of TB scheme is only able to smooth out the external bursty property of the input traffic while its internal property of self-similar pattern still remain unexplained. The self-similar property must be handled in different manner, which a statistical tool may suitably be used. Therefore, the TB scheme must also be equipped with a set of non-standard TB parameters that takes into account the performance effects of these parameters when they get involved in routing decision process. Figure 3 represents a system model of a router node, where routing decisions are to be made at the router node, based on certain decision criteria, to select the best possible forwarding link from the available outgoing links, whilst meeting the QoS demands of the application. Based on this routing model, routing decisions are to be simulated. To support the need to include the non-standard TB parameters for QoS routing decisions, the TB is proposed to work together with a Transmission Controller (TC). As standard TB can only provide basic $\mathrm{TB}$ parameters, hence $\mathrm{TC}$ is expected to provide the necessary non-TB parameters. In summary, the routing decisions are to be made by the combined function of Token Bucket (TB) and Transmission Controller (TC). 


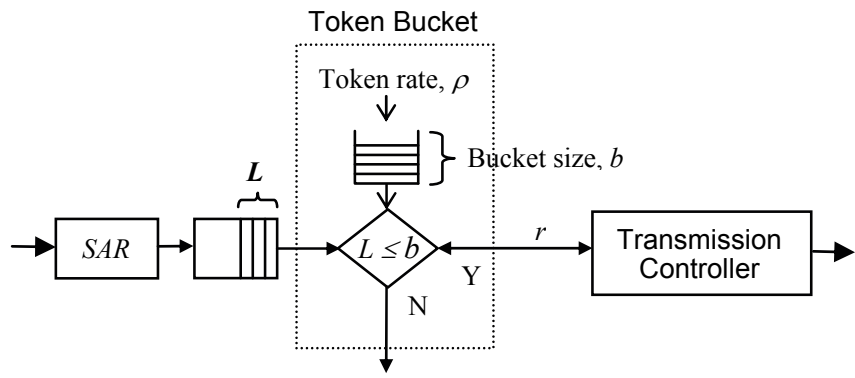

Fig. 3. The system model of a router node in Bluetooth scatternet

At the input point before submission to TB, the Segmentation and Reassembly (SAR) protocol accepts frames from upper layer and segments them into smaller packets of DHx or DMx. The types of packet produced will be determined by the SAR algorithm, which in this work Best-Fit algorithm was chosen. These packets are then put in a buffer queue with length $L$, where the queue management is a simple first-in-first-out (FIFO). A packet of size $L$ can only proceed with transmission when there is a bucket size $b$ to carry the packet, i.e. $L \leq b$. On the other hand, if $L>b$, the bucket size cannot accommodate the packet size, thus discarded from the system. When this happened, there exists packet loss probability. In a system with bursty traffic source, this loss probability is allowed to occur but to a minimum with proper control mechanism.

TB is used together with TC to produce a traffic-descriptor, which then will be used for resources reservation for QoS routing decisions. Simply, a traffic-descriptor describes the QoS requirement of the input traffic. The main function of a TB is to make routing decisions. However, TB is only parameterized with its basic parameters of $\rho$ (token rate), $b$ (bucket size), $p$ (peak rate), $m$ (minimum controlled unit), and $M$ (maximum packet size), as described by (Glasmann et al., 2000). Therefore, in this work, TC is proposed to work with TB for the reason that the basic parameters of TB are not sufficient to completely describe the QoS requirements if other decision criteria are to be considered in the decision model.

The fundamental function of a TC is to control the transmission: if the QoS requirement of the traffic is fulfilled against the available resources, forwarding of packet data over a link is allowed; else select the other links (if available). If other links are not found, just use the only single available link, probably with QoS adaptation. When adaptation is required, for instance when the available resources are not sufficient to fulfill the traffic and QoS requirements, TC will send feedback to $\mathrm{TB}$, by which necessary adjustment is then executed.

\subsection{The mathematical model}

Based on the system model and the requirement to handle the self-similar input traffic with Pareto distribution $(\alpha, k)$, according to $(\mathrm{Li}, 2002)$, the probability that a packet will have a size of length $L>b$ is

$$
p=P(L>b)=\int_{b}^{\infty} f(x) d x=\int_{b}^{\infty} \frac{\alpha k^{\alpha}}{x^{\alpha+1}} d x=(k / b)^{\alpha}
$$


where $f(x)$ is the pdf for packet size $L, \alpha$ is the shape parameter $(\alpha>1)$, and $k$ is the scale parameter that limits the $b$ value. This equation can also be interpreted as packet lost probability, i.e. the probability a packet will be discarded. It is observed that $p$ is a function of $b$. When a graph of $p$ versus $b$ is plotted, a hyperbolic graph with slow decaying rate is obtained, and it goes to infinity.

On the other hand, transmission delay $d$ experienced by a packet from this router node to the next node over a selected forwarding link is expressed by (Garroppo et al., 2001) as

$$
d=b / r
$$

where $r$ is the bit rate of the forwarding link. Since, each packet type of DHx or DMx is having its own maximum bit rate, then $r$ is assumed to have the appropriate bit rate for the transmitted packet. As can be seen, $d$ is directly related to TB via $b$ but indirectly related to TB via $r$, since $r$ is not a basic TB parameter. In this case, $r$ is to be obtained from the TC, which is a non-TB parameter. When a graph of $d$ versus $b$ is plotted, a straight-line graph is obtained.

The mathematics of algebra is then used to derive the $(r, b)$ pair, which is known as a trafficdescriptor. This pair value is the intersection point between the graphs of hyperbolic and straight line, as a result of equating Equation (6) to Equation (7). Given $r$, the required bucket size can be computed as

$$
b=\left(r k^{\alpha}\right)^{1 /(1+\alpha)}
$$

Therefore, by taking into account the bit rate of the forwarding link suitable for transmission of a specific packet type, the QoS routing decisions can now be expected to be more efficient and reliable. The $(r, b)$ pair could also be interpreted as optimal routing solution considering the various application requirements and the limited Bluetooth resources.

However, in order to provide a much better level of QoS routing decisions at a router node, further improvement could be made on the above mathematical model. In this work, specific characteristics of the source traffic and the quality of the forwarding link are further investigated. By doing so, improved efficiency and reliability for the QoS routing decisions can be expected.

One of the interesting characteristics from the source traffic is the efficiency of Bluetooth packet. The packet's efficiency is stated in (Kim et al., 2001) as $\varepsilon=\varphi /((\xi+1) \times \delta$ ), where $\varphi$ is maximum bit number for a packet type, $(\xi+1)$ is the number of slot for a single packet inclusive its acknowledgment slot, and $\delta$ is the length of a slot in bit. The efficiency of each packet type has been computed and tabulated in Table 1 .

\begin{tabular}{|c|c|}
\hline Packet type & Efficiency, $\boldsymbol{\varepsilon}$ \\
\hline DH5 & 0.72 \\
\hline DM5 & 0.48 \\
\hline DH3 & 0.59 \\
\hline DM3 & 0.39 \\
\hline DH1 & 0.17 \\
\hline DM1 & 0.10 \\
\hline
\end{tabular}

Table 1. Bluetooth packet efficiency values 
Further improvement on efficiency and reliability of QoS routing can also be achieved from the quality of the outgoing link. Typically, the quality of a forwarding link can be measured from its PER value, which is determined by the packet type forwarded on that link. They are stated by (Chen et al., 2004) respectively as follows for different packet types,

$$
\begin{gathered}
\text { PER }=1-(1-B E R)^{\mathrm{s}} \quad \text { for DHx } \\
\text { PER }=1-\left((1-B E R)^{15}+15 \times B E R \times(1-B E R)^{14}\right)^{\mathrm{s} / 15} \quad \text { for DMx }
\end{gathered}
$$

where $s$ is the maximum packet size (user payload) in bit unit, and BER is the bit error rate of the link. It is assumed that a router node has the ability to measure the BER of each of its outgoing links. In Bluetooth network, the BER value shall not be greater than $10^{-3}$ for good signal reception. Taking into account the packet efficiency and the channel quality, the effective bit rate $R(X)$ as derived by (Kim et al., 2001) for DHx or DMx packets can now be expressed as

$$
R(X)=(1-P E R(X)) \times \varepsilon \times \psi
$$

where $\psi$ is the nominal bit rate provided by a Bluetooth network, which is 1 Mbps. Substituting Equation (8) by Equation (11), the bucket size is obtained as

$$
b=\left[(1-\operatorname{PER}(X)) \times \varepsilon \times \psi \times k^{\alpha}\right]^{1 /(1+\alpha)}
$$

From Equation (11) and Equation (12), a new traffic-descriptor of the form $(R(X), b)$ is now produced. This traffic-descriptor not only takes into account the properties of the source traffic (i.e. the self-similarity and packet efficiency), but also the quality of the forwarding link (i.e. BER). With these deterministic set of information, network resources are reserved and allocated to the requesting application during a routing decision based on true scenario of the network environment. In this way, much more efficient and reliable QoS routing decisions could be made at each of the router node in the scatternet topology. Very similar to the $(r, b)$ attainment discussed previously, the $(R(X), b)$ traffic-descriptor could also be interpreted as providing only an optimal solution to the routing function, but with more accuracy due to additional parameters being added into it.

\subsection{The source traffic}

There are three types of traffic normally used by researchers in the study of traffic engineering: on-line experimental traffic, generated traffic, or video traces. In this work, video traces are chosen for the reason that they are readily available for on-line simulation, by which the frame size is directly segmented into packet counts. More importantly, the traffic have been identified by (Beran, 1994) to contain MPEG encoded data, which they are bursty and proved to associate with self-similar property. The other traffic types however, may require some forms of conversion before packet counts is produced and hence, introduced routing and transmission delays.

Specifically, the bursty traffic source is to be generated from Jurassic Park and Soccer video traces. These two traces, as well as many other traces, can be obtained publicly from website of http://www-tkn.ee.tuberlin.de/research/trace.trace.html. Each trace contained a set of frame numbers, and each frame number has its own frame size (in byte). Both traces have 
the same frame number of 89,998 but each frame has different byte length. Therefore, there is always a chance for the two traces to be different, particularly with respect to the number of packets that they may produce when the SAR segmentation algorithm is applied on each of the frame.

\subsection{The simulation}

The performance of the proposed traffic-descriptor needs to be measured to prove its ability in providing support to efficient and reliable QoS routing decision in Bluetooth ad hoc network. For this purpose, a Matlab simulation is developed to simulate the decisionmaking process made by a router node to select only one forwarding link from a set of available links, which is the optimal link. A complete route consists of a number of links, where each link is connecting two adjacent nodes, and finally a route is connecting a sender node at one end to a receiver node at the other end. Hence, routing information stored at each router node will be collected and summarized to represent the overall routing decision over a complete route. This complete route shall reflect an optimal route.

Figure 4 depicted a simulation area of $100 \mathrm{~m} \times 100 \mathrm{~m}$ where 300 nodes were randomly deployed within the area. At any point of time, a route will be established between a sender node and a receiver node over the scatternet topology. However, the success for link and route creation will be very much dependent on node density and class of transmits power of the node. The higher node density and the longer coverage radius resulted in a better chance for successful link and route creation. In this simulation, each node is assumed to use 10 meters transmit power, and for simplicity, a route is developed from left to right with fix lower left position is the sender node position and random right position is the receiver node position.

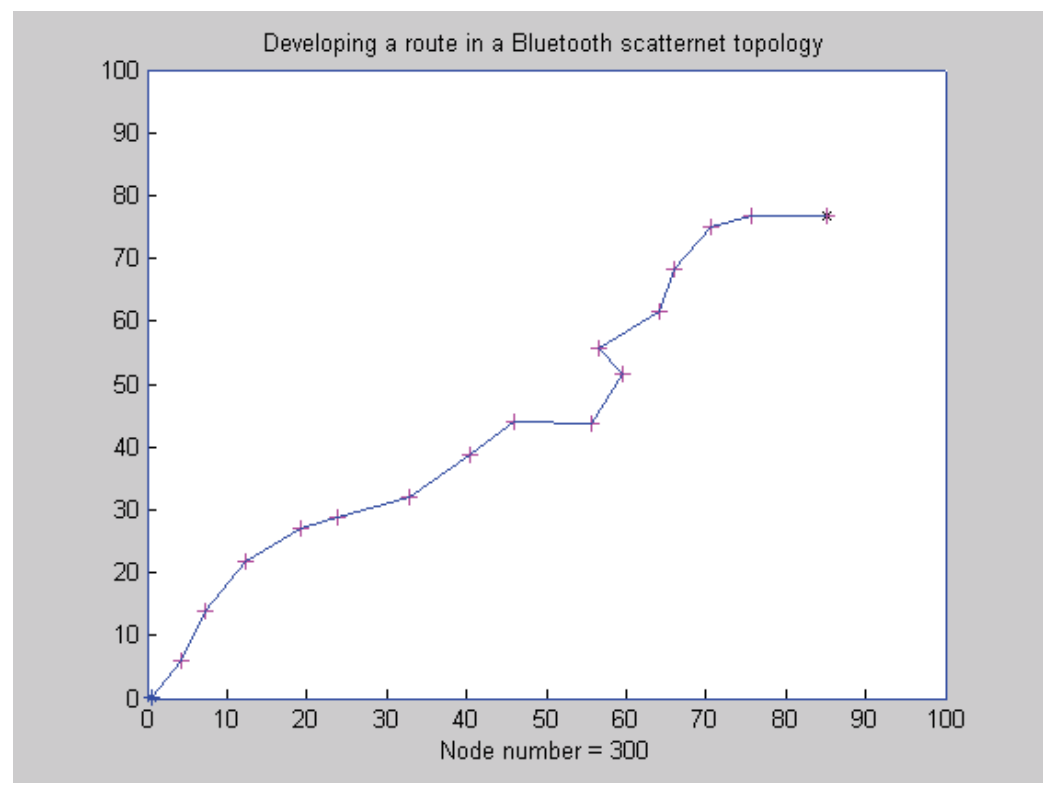

Fig. 4. Screenshot for a route creation connecting a sender and a receiver in a scatternet 
The simulation will capture the two important features of the bursty traffic: burstiness level $\alpha$ and degree of self-similarity $H$. As elaborated by (Leland et al., 1994), the relationship between them for Pareto distribution is expressed as $H=(3-\alpha) / 2$. The $H$ value will be obtained from QQ-plot method for the series of packets that is received by the router node. The QQ-plot is a statistical method used to show the level of self-similarity in a burst, and was well explained by (Dinh et al., 1998). Having the $H$ value, $\alpha$ value is then computed. Video traces of Jurassic Park and Soccer are the source of the bursty traffic. Simulation is repeated for a set of different frame ranges: say, starts at 1 to 5,000 as the smallest range size and ends at 1 to 89,998 frame number as the largest range size. Hence, the frame size is getting bigger and as well the time taken to complete the simulation is getting longer for each simulation run. This may produce different $H$ values with better accuracy. The collected $H$ values from each frame range are then averaged out and use to compute the $\alpha$ value for that frame range and for each of the video trace.

Figure 5 illustrates the implementation of Best-Fit algorithm of the SAR protocol as proposed by (Das et al., 2001). The protocol is used to segment the received bursty stream into smaller DHx or DMx packet sizes. This algorithm is executed at each router node, and total number of packets types of DHx and DMx were produced. However, the Best-Fit algorithm has the tendency to produce packets with higher capacity (such as DH5 and DH3) than to produce packets with lower capacity. For this reason, the simulation only takes DH5 packet type, since it offers higher capacity as compared to the other packet types. This simplicity is expected not to affect the performance of the proposed traffic-descriptor if lower capacity packet types are used.

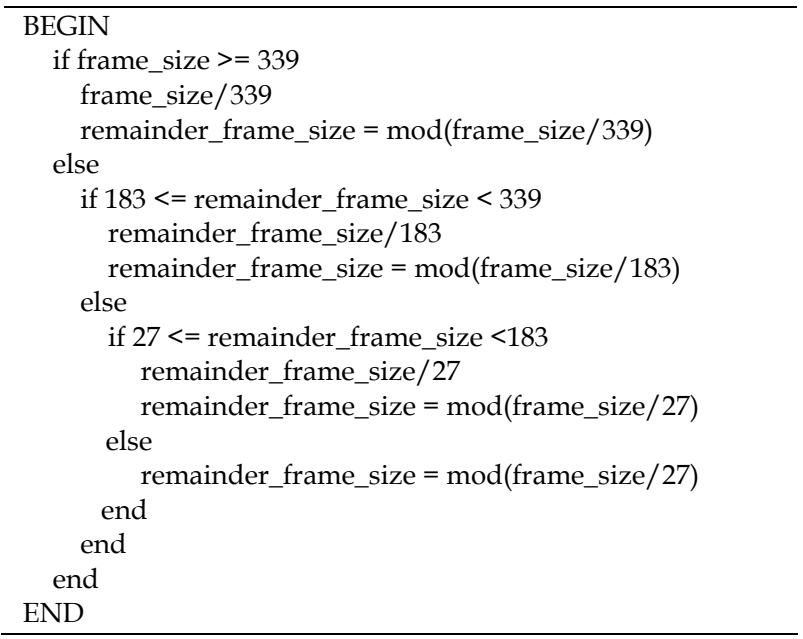

Fig. 5. The SAR Best-Fit algorithm

\section{Results and analysis}

Based on the system model described in Section 3.1 and the mathematical model in Section 3.2, Table 2 is presented to show the simulation results for the pair of traffic-descriptor of 
$(R(X), b)$ when DH5 packet is used in making routing decision at a router node. The $\alpha$ value is calculated from $H=(3-\alpha) / 2$, by which $H$ value can be obtained from the QQ-plot method for every frame range of Jurassic Park and Soccer video traces.

Five observations could be made from Table 2 and the resultant graph of Figure 6. These observations reflecting the ability of the proposed traffic-descriptor for providing support to QoS routing function in the Bluetooth ad hoc network. They are explained as follows.

\begin{tabular}{|c|c|c|c|c|}
\hline \multirow{2}{*}{ Frame range } & \multicolumn{2}{|c|}{$\alpha$} & \multicolumn{2}{c|}{$(\boldsymbol{R}(X), \boldsymbol{b})$} \\
\cline { 2 - 5 } & Jurassic & Soccer & Jurassic & Soccer \\
\hline $1-5,000$ & 0.910 & 0.911 & 719374,30870 & 719379,30887 \\
\hline $1-10,000$ & 0.918 & 0.918 & 718976,30384 & 718981,30395 \\
\hline $1-15,000$ & 0.926 & 0.927 & 718931,30124 & 718928,30134 \\
\hline $1-20,000$ & 0.937 & 0.935 & 719381,29849 & 719389,29850 \\
\hline $1-25,000$ & 0.952 & 0.953 & 718714,29051 & 718720,29048 \\
\hline $1-30,000$ & 0.967 & 0.968 & 719116,28323 & 719121,28332 \\
\hline $1-35,000$ & 0.983 & 0.983 & 719177,27552 & 719170,27565 \\
\hline $1-40,000$ & 0.994 & 0.994 & 719356,27047 & 719358,27053 \\
\hline $1-45,000$ & 1.001 & 1.002 & 718025,26721 & 718029,26717 \\
\hline $1-50,000$ & 1.008 & 1.009 & 719300,26453 & 719296,26459 \\
\hline $1-55,000$ & 1.012 & 1.012 & 719196,26267 & 719200,26273 \\
\hline $1-60,000$ & 1.019 & 1.018 & 715380,25927 & 715384,25930 \\
\hline $1-65,000$ & 1.024 & 1.025 & 718904,25778 & 718900,25781 \\
\hline $1-70,000$ & 1.028 & 1.029 & 714636,25510 & 714643,25507 \\
\hline $1-75,000$ & 1.032 & 1.031 & 718406,25450 & 718410,25459 \\
\hline $1-80,000$ & 1.034 & 1.035 & 719346,25349 & 719349,25354 \\
\hline $1-85,000$ & 1.037 & 1.037 & 715426,25189 & 715433,25196 \\
\hline $1-89,998$ & 1.038 & 1.039 & 719136,25205 & 719138,25200 \\
\hline
\end{tabular}

Table 2. The effect of burst on $(R(X), b)$ for DH5 packet transmission

First, traffic-descriptors of $(R(X), b)$ for Jurassic Park and Soccer have been produced. This pair values provides the requesting application with information, which can be communicated to the Resource Manager for reservation and allocation of resources. The pair is a parsimonious traffic-descriptor since it contained only least number of parameters but has the ability to describe the required resources accurately for QoS routing decision in the Bluetooth ad hoc network. However, depending on the frame range and the number of packets they produced after segmentation, the resulting values of $(R(X), b)$ may differ from each other.

Second, as can be observed from Table 2, while $R(X)$ values have changed randomly and not following a certain order, $b$ values reduced linearly as $\alpha$ values go higher. This is graphically presented in Figure 6, where both video traces have shown similar graph pattern. 
Importantly, the $R(X)$ values are practical values that fluctuate within the acceptable range and come close to the bit rate of 723,200 bps for DH5 packet.

Third, the accuracy of the traffic-descriptor $(R(X), b)$ can be measured from the values of effective bit rate $R(X)$. The mean value for $R(X)$ is 718,380 bps for both Jurassic Park and Soccer. When this is compared against the maximum allowable bit rate of 723,200 bps for DH5 packet as specified in the (Bluetooth Specification v1.0B, 1999), the mean value is clearly within an acceptable range. The difference between them is only $4,820 \mathrm{bps}$, which is equivalent to $0.66 \%$. In particular, after taking into account the channel quality at the time when forwarding decision was made at a router node, this $R(X)$ value can be considered as a realistic value suitable for practical implementation.

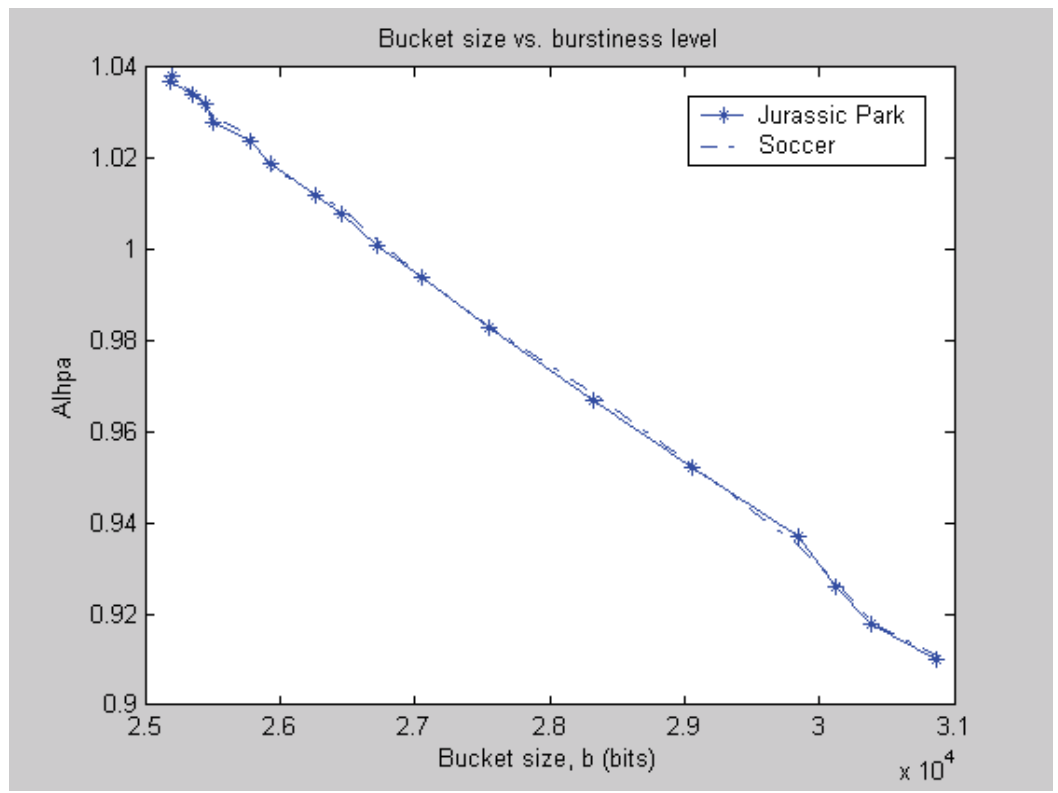

Fig. 6. Burstiness level $\alpha$ versus bucket size $b$ for Jurassic Park and Soccer video traces

Forth, when $\alpha$ value is deviating away from 1 but approaching to 2 (which implies a less bursty traffic), the bucket size gets smaller. It means that the lower variability in the source traffic, the smaller required bucket size in handling the burst. Subsequently, the smaller bucket size the better routing performance, in term of its transmission delay from the queue. Therefore, $\alpha \rightarrow 2$ (or equivalently $H \rightarrow 0.5$ ) is very much needed. In this way, traffic with lower burst level could be produced, by which the performance of a QoS routing control scheme could be more effective and reliable. This is critically needed when limited link (or only a single link) and limited resources of Bluetooth network are available, but to provide a level of service guarantee to the requesting applications.

Fifth, for both video traces, bucket size can be used to control the burstiness level of the input traffic. It can be observed from the graph that bigger bucket size would imply lower variability in the input traffic, and vice-versa. However, careful must be taken to allow only 
suitable bucket size to operate, i.e. too big bucket size may lead to significantly higher packet lost probability, thus may lead to system collapse. Therefore, it is important to balance between the bucket size to use and the allowable burstiness level for efficient and reliable QoS routing decision at a router node.

\section{Conclusions and future work}

A traffic-descriptor $(r, b)$ of which contained non-Token-Bucket parameters has been successfully developed. In particular, the developed traffic-descriptor has parsimoniously described the application requirement to the Resource Manager for the required resources. The model has been parameterized with only least number of parameters, but it has the capability to describe very well the QoS routing requirement. Additionally, to achieve better level of service guarantee in routing, $(R(X), b)$ traffic-descriptor has been developed as well. It has dimensioned the parameters of the input traffic and the channel quality using both standard Token-Bucket and non-Token-Bucket parameters for much more accurate resource reservation and allocation. Hence, the QoS routing decisions at a router node are then made to be more efficient and reliable. However, this traffic-descriptor is only applicable to Bluetooth ad hoc network setting. This is because the traffic-descriptor has characterized the source traffic and the channel quality by mean of a mathematical model that is dependent on Bluetooth network setting.

In the case of Bluetooth's DH5 packet transmission, it was found that the traffic-descriptor has accurately described the required effective bit rate $R(X)$ to only a difference of $0.66 \%$ from its maximum bit rate, whilst QoS guarantee is granted to user application. Therefore, the developed mathematical model is assumed to represent the system correctly and to work accordingly. The final result shall be exhibited through the optimal usage of the network resources. This is critically needed as to achieve efficient and reliable routing decision-making processes at the router nodes, since Bluetooth network is having only a handful of resources.

Therefore, the contribution of this work was in the development of a parsimonious trafficdescriptor that has incorporated the non-Token-Bucket parameters, in addition to basic Token-Bucket parameters, through the formulation of a mathematical model that is suitable only for use in Bluetooth ad hoc network. Hence, the identified future work is to develop a general-purpose traffic-descriptor, which can be used to dimension any other non-TokenBucket parameters for use in any types of wireless networks.

\section{References}

A. Das, A. Ghose, A. Razdan, H. Saran \& R. Shorey, (2001). Enhancing performance of asynchronous data traffic over the Bluetooth wireless ad hoc network. Proceedings of the 20 $0^{\text {th }}$ Annual Joint Conference of IEEE Computer $\mathcal{E}$ Communications Society (INFOCOM 2001), pp. 591-600.

Bluetooth Specifications v1.0B, (1999). Bluetooth SIG. Available at: http://www.bluetooth.com, [Accessed on 2 August 2010]. 
F.Y. Li, (2002). Local and global QoS-aware Token Bucket parameters determination for traffic conditioning in $3^{\text {rd }}$ generation wireless networks. Proceedings of the European Wireless'02, pp. 362-368.

G. Procissi, M. Gerla, J. Kim, S.S. Lee \& M.Y. Sanadidi, (2001). On long range dependence and Token Buckets. Proceedings of the SPECTS'01.

IEEE 802.15 Specifications. Available at http://standards.ieee.org/getieee802/802.15.html, [Accessed on 2 August 2010].

J. Beran, (1994). Statistics for Long-Memory Processes, Chapman and Hall/CRC, $1^{\text {st }}$ edition, New York.

J. Glasmann, M. Czermin \& A. Riedl, (2000). Estimation of Token Bucket parameters for videoconferencing systems in corporate networks. Proceedings of the International Conference on Software, Telecommunications and Computer Networks.

J. Haartsen, W. Allen, J. Inouye, O.J. Joeressen \& M. Naghshineh, (1998). Bluetooth: vision, goals and architecture. ACM Mobile Computing and Communications Review, Vol. 1, No. 2, pp. 1-8.

J. Kim, Y. Lim, Y. Kim \& J.S. Ma, (2001). An adaptive segmentation scheme for the Bluetooth-based wireless channel. Proceedings of the IEEE IC3N' 01 , pp. 440-445.

K. Park, G. Kim \& M. Crovella, (1996). On the relationship between file sizes, transport protocols, and self-similar network traffic. Proceedings of the IEEE International Conference Network Protocols, pp. 171-180.

L.J. Chen, R. Kapoor, M.Y. Sanadidi \& M. Gerla, (2004). Enhancing Bluetooth TCP throughput via link layer packet adaptation. Proceedings of the IEEE International Conference on Communications'04.

M. Taqqu, W. Willinger \& R. Sherman, (1997). Proof of a fundamental result in self-similar traffic modeling. ACM/SIGCOMM Computer Communications Review, Vol. 27, pp. 523.

M.D. Norashidah \& F. Norsheila, (2007). Fuzzy logic Token Bucket bandwidth predictor for assured forwarding traffic in a DiffServ-aware MPLS Internet. Proceedings of the Asia International Conference on Modelling \& Simulation (AMS'07).

M.E. Crovella \& A. Bestavros, (1999). Self-similarity in world wide web traffic: evidence and possible causes. IEEE/ACM Transactions on Networking, Vol. 5, No. 6, pp. 835-846.

M.E. Crovella \& L. Lipsky, (1997). Long-lasting transient conditions in simulations with heavy-tailed workloads. Proceedings of the Winter Simulation Conference, pp. 10051012.

R. Handle, M. Anber \& S. Schroder, (1996). ATM Networks Concepts, Protocols and Applications, Addison-Wesley, New York.

R.G. Garroppo, S. Giordano, S. Niccolini \& F. Russo, (2001). A simulation analysis of aggregation strategies in $\mathrm{WF}^{2} \mathrm{Q}+$ schedulers network, IP Telephony'01.

S. Fernandes, C. Kamienski \& D. Sadok, (2003). Accurate and fast replication on the generation of fractal network traffic using alternative probability models. Proceedings of the SPIE 5244, pp. 154-163.

S. Valaee \& J-C. Gregoire, (2005). An estimator of regulator parameters in a stochastic setting. IEEE/ACM Transaction on Networking, Vol. 13, No. 6, pp. 1376-1389. 
T.D. Dinh, S. Molnar \& A. Vidacs, (1998). Investigation of fractal properties in data traffic. Journal of Communications, Vol. XLIX, pp. 12-18.

V. Paxson, (1994). Empirically derived analytic models of wide area TCP connections. IEEE/ACM Transactions on Networking, Vol. 2, No. 4, pp. 316-336.

W.E, Leland, M.S. Taqqu, W. Willinger \& D.V. Wilson, (1994). On the self-similar nature of Ethernet traffic (extended version). IEEE/ACM Transactions on Networking, Vol. 2, No. 1, pp. 1-15.

X. Yang, (2000). Designing traffic profiles for bursty Internet traffic. Proceedings of the IEEE Global Internet, pp. 2149-2154.

Z. Hadzi-Velkov \& L. Garrilovska, (1999). Performance of the IEEE802.11 wireless LANs and influence of hidden terminals. Proceedings of the Telsiks'99, pp. 102-105. 


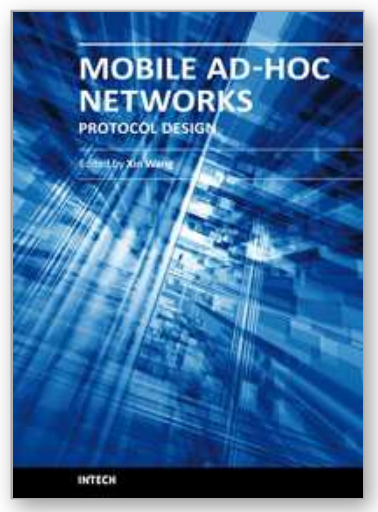

\author{
Mobile Ad-Hoc Networks: Protocol Design \\ Edited by Prof. Xin Wang
}

ISBN 978-953-307-402-3

Hard cover, 656 pages

Publisher InTech

Published online 30, January, 2011

Published in print edition January, 2011

Being infrastructure-less and without central administration control, wireless ad-hoc networking is playing a more and more important role in extending the coverage of traditional wireless infrastructure (cellular networks, wireless LAN, etc). This book includes state-of-the-art techniques and solutions for wireless ad-hoc networks. It focuses on the following topics in ad-hoc networks: quality-of-service and video communication, routing protocol and cross-layer design. A few interesting problems about security and delay-tolerant networks are also discussed. This book is targeted to provide network engineers and researchers with design guidelines for large scale wireless ad hoc networks.

\title{
How to reference
}

In order to correctly reference this scholarly work, feel free to copy and paste the following:

Halabi Hasbullah and Mahamod Ismail (2011). The Dimensioning of Non-Token-Bucket Parameters for Efficient and Reliable QoS Routing Decisions in Bluetooth Ad Hoc Network, Mobile Ad-Hoc Networks: Protocol Design, Prof. Xin Wang (Ed.), ISBN: 978-953-307-402-3, InTech, Available from:

http://www.intechopen.com/books/mobile-ad-hoc-networks-protocol-design/the-dimensioning-of-non-tokenbucket-parameters-for-efficient-and-reliable-qos-routing-decisions-in-

\section{INTECH}

open science | open minds

\section{InTech Europe}

University Campus STeP Ri

Slavka Krautzeka 83/A

51000 Rijeka, Croatia

Phone: +385 (51) 770447

Fax: +385 (51) 686166

www.intechopen.com

\section{InTech China}

Unit 405, Office Block, Hotel Equatorial Shanghai

No.65, Yan An Road (West), Shanghai, 200040, China 中国上海市延安西路65号上海国际贵都大饭店办公楼 405 单元

Phone: +86-21-62489820

Fax: $+86-21-62489821$ 
(C) 2011 The Author(s). Licensee IntechOpen. This chapter is distributed under the terms of the Creative Commons Attribution-NonCommercialShareAlike-3.0 License, which permits use, distribution and reproduction for non-commercial purposes, provided the original is properly cited and derivative works building on this content are distributed under the same license. 